\title{
Rehabilitation Recommendations for Multiple Sclerosis Patients during the COVID-19 Pandemic
}

\author{
Abdorreza Naser Moghadasi, MD; Mohaddeseh Azadvari, MD ${ }^{1,2^{*}}$; Mohammad Ali Sahraian, MD \\ ${ }^{1}$ Multiple Sclerosis Research Center, Neuroscience Institute, Tehran University of Medical Sciences, Tehran, Iran \\ ${ }^{2}$ Physical Medicine and Rehabilitation Department, Tehran University of Medical Sciences, Tehran, Iran
}

\section{Dear Editor,}

The COVID-19 disease, which is caused by the new coronavirus, is a contagious illness that causes respiratory problems, physical disabilities, and psychological problems. ${ }^{1,2}$ All people in the community, including multiple sclerosis (MS) patients, are at risk for the COVID-19 disease. It is more important in MS patients because these patients may be at increased risk for more severe disease due to their medications. Continuous rehabilitation is an essential need for MS patients because of their symptoms including spasticity, fatigue and others. ${ }^{3-5}$ In the coronavirus pandemic situation and the quarantine period, the patients do not have access to equipped rehabilitation centers. So, special attention should be paid to the rehabilitation program of these patients. The patients with COVID-19 need respiratory rehabilitation and MS patients with COVID-19 need more rehabilitation programs including respiratory, physical and digestive rehabilitation. Considering the highly contagious coronavirus, rehabilitation specialists must prepare educational material about exercises and rehabilitation in videos, brochures etc. There is currently little information available about the standard rehabilitation of the COVID-19 disease in MS patients, but we can recommend the following in this regard:

\section{Recommendation for MS Patients without COVID-19} Non-disabled Patients (Expanded Disability Status Scale $<6$ )

- We recommend regular physical activities as possible such as walking in room and cycling on a stationary bike, balanced diet, enough rest and quitting smoking.

- Routine exercise including: active or active assisted range of motion (ROM) exercise for all joints twice a day every day; active or active assisted stretching exercise twice a day, every day especially for calf and hamstring muscles and shoulder region and pectoral muscles; resistance exercise twice per week, 10-15 repetitions.
- Digestive rehabilitation: constipation is one of the common problems in MS patient that may be worsened by decreased mobility during the coronavirus pandemic. They must drink enough liquids and have enough physical activity such as walking in room.

Disabled Patients (Expanded Disability Status Scale $\geq 6$ )

- Routine exercise including: bed rolling, change of position, contraction of abdominal, gluteal, thigh and ankle muscles frequently, every day. Passive ROM exercise for all joints twice a day, every day. Passive stretching exercise twice a day, every day especially for spastic muscles.

- Digestive rehabilitation: In addition to enough liquid intake, they must move in bed or wheelchair frequently, and perform anal lifting and contraction of the abdominal muscles to improve blood circulation in the abdomen and improve digestive function. ${ }^{3}$

\section{Recommendation for MS Patients with Mild COVID-19} In addition to the pervious exercise, they must do the following exercise depending on their general and pulmonary conditions under a physician's supervision: Breathing exercises: Deep breathing and diaphragmatic breathing exercises, pursed lip breathing, coughing with the Huff technique, twice a day for at least ten minutes. ${ }^{6}$ Exercise for deep vein thrombosis prevention: Active or passive ankle pumping and ankle rotation at least twice a day, recurrent tightening and relaxing leg and thigh muscles.

Full body exercise: Stepping in the air, arch bridge, and stretching sit-ups as possible for non-disabled patients. ${ }^{6}$

The patients should stop exercising as soon as one of the following conditions occurs, and seek doctor's help: Dyspnea, chest tightness, dizziness, headache, blurred vision, palpitations, sweating, inability to maintain balance, etc. ${ }^{2}$ 


\section{Recommendation for MS Patients with Severe COVID-19}

In these patients, a comprehensive assessment of the overall functional status of the patient must be performed; if the patient is stable, rehabilitation by an expert therapist must be started as soon as possible.

In general, it can be recommended that MS patients need more attention for rehabilitation in the COVID-19 pandemic. These patients may experience more anxiety about the coronavirus than common people; so, psychological interventions and reassurance can be helpful and necessary. For optimal management of MS patients in this pandemic situation, physicians and rehabilitation teams must be available to patients and do the most for remote rehabilitation education.

\section{Authors' Contribution}

ANM and MA wrote the manuscript and provided data. MAS supervised the manuscript. All authors reviewed the final manuscript.

\author{
Conflict of Interest Disclosures \\ None.
}

\section{Ethical Statement}

Not applicable.

\section{References}

1. Wang C, Horby PW, Hayden FG, Gao GF. A novel coronavirus outbreak of global health concern. Lancet. 2020;395(10223):470-3. doi: 10.1016/S01406736(20)30185-9.

2. 2019 New Coronary Virus Pneumonia Respiratory Rehabilitation Guidance (Second Edition). Chin J Tubere Respir Dis. 2020. doi: 10.3760/cma.j.cn112147-20200228-00206.

3. Anjali Shah AF, Nourbakhsh B, Stüve O. Multiple Sclerosis. In: Cifu DX, ed. Braddom's Physical Medicine \& Rehabilitation. 5th ed. Philadelphia, PA: Elsevier. 2016: 1029-59.

4. Awad RA. Neurogenic bowel dysfunction in patients with spinal cord injury, myelomeningocele, multiple sclerosis and Parkinson's disease. World J Gastroenterol. 2011;17(46):503548. doi: 10.3748/wjg.v17.i46.5035.

5. Barnes MP, Kent RM, Semlyen JK, McMullen KM. Spasticity in multiple sclerosis. Neurorehabil Neural Repair. 2003;17(1):6670. doi: $10.1177 / 0888439002250449$.

6. Yang F, Liu N, Hu JY, Wu LL, Su GS, Zhong NS, et al. Pulmonary rehabilitation guidelines in the principle of $4 \mathrm{~S}$ for patients infected with 2019 novel coronavirus (2019-nCoV). Zhonghua Jie He He Hu Xi Za Zhi. 2020;43(3):180-2. doi: 10.3760/cma.j.issn.1001-0939.2020.03.007.

Received: April 3, 2020, Accepted: April 5, 2020, ePublished: July 1, 2020

Cite this article as: Moghadasi AN, Azadvari M, Sahraian MA. Rehabilitation recommendations for multiple sclerosis patients during the COVID-19 pandemic. Arch Iran Med. 2020;23(7):509-510. doi: 10.34172/aim.2020.51.

(c) (c) (c) 2020 The Author(s). This is an open-access article distributed under the terms of the Creative Commons Attribution License (http://creativecommons. org/licenses/by/4.0), which permits unrestricted use, distribution, and reproduction in any medium, provided the original work is properly cited. 